\title{
Current strategies for coping with the hematocrit problem in dried blood spot analysis
}

\author{
"The hematocrit problem is the most widely discussed issue \\ in dried blood spot analysis."
}

\begin{abstract}
Keywords: dried blood spots $\bullet$ dried plasma spots $\bullet$ hematocrit effect $\bullet$ hematocrit prediction • partial-spot analysis $\bullet$ special filter substrates $\bullet$ whole-spot analysis
\end{abstract}

The hematocrit (Hct) problem is the most widely discussed issue in dried blood spot (DBS) analysis. As Hct determines the viscosity of blood, it affects the spreading of blood spotted on filter paper. Therefore, partial punches taken from DBS prepared from blood with different Hct values will contain different amounts of blood. Hence, depending on the analyte of interest and the filter paper used, deviating Hct values may cause significant assay bias. Hct may additionally influence the potential bias introduced by the site of punching, the recovery of a particular compound from DBS and/or have an impact on matrix effects. As a consequence, the Hct effect is generally recognized as the most important factor hampering the widespread application of DBS [1-3]. Therefore, although not yet common practice, it is advisable to include quality control samples prepared from blood with different Hct values in validation experiments in order to define a Hct interval in which the obtained DBS results are still valid [4]. In addition, to minimize the impact of Hct, calibrators should be prepared using blood with a Hct value close to the expected median of the target population. However, even when a Hct interval has been defined in which the assay bias is within acceptable limits (typically $\pm 15 \%$ ), one still needs to be able to verify whether the Hct of a given DBS actually lies within this interval. Furthermore, in the comparison between concentrations measured in DBS and plasma or serum, the Hct of the blood used to prepare the DBS is an important factor as well.
In the last few years, several strategies have been proposed to cope with the Hct problem in DBS analysis. These strategies focus on overcoming the issue of differential spreading of blood on filter paper, as this is generally considered to be the major contributor to Hct-induced bias. The influence of Hct on recovery and the matrix effect, on the other hand, may be minimized by optimizing the extraction or chromatographic conditions [5]. For example, Ooms et al. demonstrated that the use of elevated temperatures in an automated flow-through desorption procedure may reduce the effect of Hct on the recovery of several drugs [6].

\section{Whole-spot analysis}

As pointed out by Fan and Lee in an editorial from 2012 published in this journal, an easy way to deal with the Hct problem is to simply analyze whole, volumetrically applied spots, instead of using fixed-size partial punches [7]. The entire DBS can be punched out after the application of a defined volume of blood or the blood can be spotted onto prepunched discs. An interesting example of the latter approach is the concept of 'dried matrix on paper discs', in which precut paper discs are placed in a dedicated support system [8]. However, an important limitation inherent to all whole-spot approaches is the need for an accurate and precise delivery of a volume of blood onto the filter paper. Although convenient tools are available to facilitate this volumetric application, such as an incremental dispenser developed by Drummond Scientific Company (PA, USA) or Micro-
Pieter MM De Kesel

Laboratory of Toxicology, Ghent University, Faculty of Pharmaceutical Sciences, Ottergemsesteenweg 460, 9000 Ghent, Belgium

\section{Sara Capiau}

Laboratory of Toxicology, Ghent University, Faculty of Pharmaceutical Sciences, Ottergemsesteenweg 460, 9000 Ghent, Belgium

\section{Willy E Lambert}

Laboratory of Toxicology, Ghent University, Faculty of Pharmaceutical Sciences, Ottergemsesteenweg 460, 9000 Ghent, Belgium

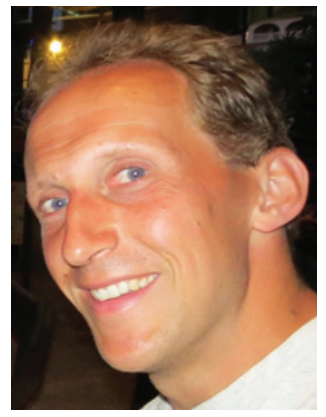

Christophe P Stove

Author for correspondence: Laboratory of Toxicology, Ghent University, Faculty of Pharmaceutical Sciences, Ottergemsesteenweg 460, 9000 Ghent, Belgium Tel.: +32 92648121 Fax: +3292648183 christophe.stove@ugent.be 
safe ${ }^{\circledR}$ pipettes (Safe-Tec Clinical Products LLC., PA, USA) [9], in our opinion, any volumetric whole-spot approach is only feasible in a specialized setting at present, where spotting is carried out by trained personnel. When capillary sampling is to be performed by nonexperienced individuals themselves (e.g., sampling at home, representing an important benefit of DBS sampling over traditional venous sampling), direct application of a drop of blood from the fingertip onto the DBS card may be preferred, provided that clear instructions on good sampling practices are given to the involved subjects. It can be envisaged that, in the near future, new developments, particularly microfluidic devices such as the 'disposable metering device' prototype developed by Lenk et al. [10] or alternative devices being developed by others [11], will allow volumetric deposition of nonvolumetrically deposited samples. Another promising approach for volumetric collection of blood to generate dried samples is the use of a very recently developed alternative collection device (VAMS - Volumetric Absorptive Microsampling), which wicks up a fixed volume of blood, independent of the Hct [12].

\section{Dried plasma spots}

Several authors reported on using dried plasma spots (DPS) instead of DBS, representing another approach for avoiding the Hct problem, while taking advantage of the ease of processing, transporting or storing of dried matrix samples. In most studies, plasma was obtained from venous whole blood after centrifugation. Preparation of plasma from microsamples of capillary blood, as applied by Jonsson et al. in a toxicokinetic study in rats [13], simplifies the sampling process, as venipuncture is no longer needed. However, this methodology still requires centrifugation, which is, similar to the aforementioned volumetric approach, only feasible in a laboratory setting. Interesting membrane filtration devices for the preparation of DPS from whole blood have been used recently [14,15]. With these devices, DPS are generated by applying drops of blood onto a multilayered extraction card, in essence consisting of a separation and a collection membrane. While the plasma can flow through the separation membrane to the bottom collection membrane, the cellular fraction is trapped at the top. After waiting for a few minutes, this top layer can be peeled off and plasma spots (of a fixed volume or not) are formed on the collection membrane. Although more studies involving different analytes and evaluating the correlation between plasma and DPS concentrations are needed in order to support the general usefulness of this approach, the application of these devices seems to be a promising strategy for overcoming the Hct problem.

\section{Special filter substrates}

Several efforts have been made in trying to reduce the Hct effect by developing special filter substrates that would be less affected by Hct. A possibly interesting development is HemaSpot ${ }^{\mathrm{TM}}$ (Spot On Sciences, Inc., TX, USA), a cartridge containing a fan-shaped filter paper with multiple blades protruding from the center. As claimed by the manufacturer, the use of this filter paper should result in a reduced Hct effect [16]. Another Hct-independent DBS card was developed by Mengerink [17]. The added-value of these materials in controlling the Hct-dependent spreading of blood remains to be established.

\section{Measure or predict the Hct}

As mentioned earlier, when the Hct of the blood originally used to prepare a DBS is known, it can be checked as to whether it is within the accepted Hct range defined during method validation. Furthermore, when a strongly deviating Hct value is found, it could be incorporated into an established algorithm that enables the correction of the analyte concentration. Obviously, the Hct can be determined in a (venous) blood sample collected along with the capillary DBS sample [7]. However, in this case, except when preparing DBS improves the compound stability, it may be easier to directly analyze the blood (micro)sample. Alternatively, the Hct can be determined in capillary blood using anticoagulant-coated capillaries [18]. Again, as this approach requires centrifugation of the capillaries, home sampling is impossible. A more practical option in this context may be to use point-of-care testing systems in order to directly measure the hemoglobin in blood, which enables the calculation of Hct [19]. It is questionable, however, as to whether equipping every patient with such a (relatively expensive) device is feasible.

\section{"While these and future developments will undoubtedly contribute to the more widespread application and acceptance of dried blood spot ... sampling, other challenges remain..."}

An alternative and convenient strategy for coping with the Hct problem while allowing patient self-sampling may be to estimate the Hct of a DBS by measuring an endogenous compound in the DBS that correlates with Hct. Potassium has been proven to be a good Hct predictor, as we previously demonstrated [20]. Potassium concentrations were measured on a routine clinical chemistry analyzer following extraction from 3-mm DBS punches, yielding a straightforward, fast, costeffective and automatable procedure. The developed method was fully validated and its application on real patient samples showed a good correlation between the 
predicted Hct and the actual Hct measured in venous blood samples. In a recent follow-up study, we could demonstrate that we were able to develop an algorithm, using the predicted Hct or even the DBS potassium concentrations alone, that allowed us to correct for the Hct bias in the DBS-based quantification of selected analytes [De Kesel PM, Capiau S, Stove VV, Lambert We, Stove CP. Potassium-BASED ALgorithm ALlOWS CORRECTION FOR THE HEMATOCRIT BIAS IN QUANTITATIVE ANALYSIS OF CAFFEINE AND ITS MAJOR metabolite in dried blood spots (Submitted, Under Review)]. While this approach is promising, broader application of this procedure is also required on more analytes in order to confirm its widespread applicability.

In conclusion, several strategies for coping with the Hct problem have been developed recently. The Hct effect may be avoided by analyzing whole DBS. This approach, requiring accurate volumetric applications of blood, is well-suited for use in a controlled setting, but is currently still difficult to implement in patient self-sampling scenarios. The latter also applies to DPS obtained by spotting plasma following the centrifugation of blood. In this context, generating DPS from drops of whole blood by using membrane filtration devices may be a promising technique. Furthermore, the impact of Hct

\section{References}

1 De Kesel PM, Sadones N, Capiau S, Lambert WE, Stove CP. Hemato-critical issues in quantitative analysis of dried blood spots: challenges and solutions. Bioanalysis 5(16), 2023-2041 (2013).

2 Denniff P, Spooner N. The effect of hematocrit on assay bias when using DBS samples for the quantitative bioanalysis of drugs. Bioanalysis 2(8), 1385-1395 (2010).

3 De Vries R, Barfield M, van de Merbel $N$ et al. The effect of hematocrit on bioanalysis of DBS: results from the EBF DBS-microsampling consortium. Bioanalysis 5(17), 2147-2160 (2013).

4 Ingels AS, De Paepe P, Anseeuw K et al. Dried blood spot punches for confirmation of suspected $\gamma$-hydroxybutyric acid intoxications: validation of an optimized GC-MS procedure. Bioanalysis 3(20), 2271-2281 (2011).

5 Youhnovski N, Bergeron A, Furtado M, Garofolo F. Pre-cut dried blood spot (PCDBS): an alternative to dried blood spot (DBS) technique to overcome hematocrit impact. Rapid Commun. Mass Spectrom. 25(19), 2951-2958 (2011).

6 Ooms B, Hempen C, Knegt L. Towards unbiased dried blood spot analysis using temperature-enhanced flowthrough desorption coupled online to solid-phase extraction and mass spectrometry. Presented at: EBF 6th Open Meeting. Barcelona, Spain, 20-22 November 2013.

7 Fan L, Lee JA. Managing the effect of hematocrit on DBS analysis in a regulated environment. Bioanalysis 4(4), 345-347 (2012).

8 Meesters RJ, Zhang J, van Huizen NA, Hooff GP, Gruters RA, Luider TM. Dried matrix on paper disks: the next may be reduced by using special filter materials in which the spreading of blood is less influenced by Hct. Finally, measuring or predicting the Hct of a DBS allows for the verification of whether Hct falls within a validated range and for the correction of the Hct-induced bias. The Hct can either be directly measured in blood or predicted based upon an endogenous parameter, such as the potassium concentration in DBS extracts. While these and future developments will undoubtedly contribute to the more widespread application and acceptance of DBS (and DPS) sampling, other challenges remain, such as the possible differences between venous and capillary concentrations, a challenge that is associated with any microsampling approach.

\section{Financial \& competing interests disclosure}

S Capiau wishes to acknowledge the FWO Research Foundation - Flanders for granting her a PhD Fellowship. The authors have no other relevant affiliations or financial involvement with any organization or entity with a financial interest in or financial conflict with the subject matter or materials discussed in the manuscript apart from those disclosed.

No writing assistance was utilized in the production of this manuscript.

generation DBS microsampling technique for managing the hematocrit effect in DBS analysis. Bioanalysis 4(16), 2027-2035 (2012).

9 Li FM, Ploch S, Fast D, Michael S. Perforated dried blood spot accurate microsampling: the concept and its applications in toxicokinetic sample collection. J. Mass Spectrom. 47(5), 655-667 (2012).

10 Lenk G, Pohanka A, Stemme G, Beck O, Roxhed N. A disposable chip enabling metering in dried blood spot sampling. Presented at: 17th International Conference on Miniaturized Systems for Chemistry and Life Sciences. Freiburg, Germany, 27-31 October 2013.

11 DBS System, Ltd. http://dbs-system.ch

12 Denniff P, Spooner N. Volumetric absorbtive micro sampling (VAMS): a novel dried sample collection technique for quantitative bioanalysis. Anal. Chem. doi:10.1021/ac5022562 (2014) (Epub ahead of print).

13 Jonsson O, Palma Villar R, Nilsson LB et al. Capillary microsampling of $25 \mu \mathrm{l}$ blood for the determination of toxicokinetic parameters in regulatory studies in animals. Bioanalysis 4(6), 661-674 (2012).

14 Li YY, Henion J, Abbott R, Wang P. The use of a membrane filtration device to form dried plasma spots for the quantitative determination of guanfacine in whole blood. Rapid Commun. Mass Spectrom. 26(10), 1208-1212 (2012).

15 Kim JH, Woenker T, Adamec J, Regnier F. Simple, miniaturized blood plasma extraction method. Anal. Chem. 85(23), 11501-11508 (2013).

16 Spot On Sciences. http://spotonsciences.com 
17 Mengerink Y. New Ht independent dried blood spot cards discussion. Presented at: EBF 6th Open Meeting. Barcelona, Spain, 20-22 November 2013.

18 Bouman LN, Bernards JA, Boddeke HWGM. Bloed: samenstelling en functies. In: Medische Fysiologie (2e Herziene Druk). (Blood: composition and functions. In: Medical Physiology (2nd Revised Edition)). Bouman LN, Bernards JA, Boddeke HWGM (Eds). Bohn Stafleu van Loghum, The Netherlands, 395-422 (2008).
19 Skelton VA, Wijayasinghe N, Sharafudeen S, Sange A, Parry NS, Junghans C. Evaluation of point-of-care haemoglobin measuring devices: a comparison of Radical-7 pulse co-oximetry, HemoCue ${ }^{\circledR}$ and laboratory haemoglobin measurements in obstetric patients. Anaesthesia 68 (1), 40-45 (2013).

20 Capiau S, Stove VV, Lambert WE, Stove CP. Prediction of the hematocrit of dried blood spots via potassium measurement on a routine clinical chemistry analyzer. Anal. Chem. 85(1), 404-410 (2013). 